\title{
Indentation by nominally flat or conical indenters with rounded corners
}

\author{
Michele Ciavarella* \\ Department of Engineering Science, Oxford University, Parks Road, Oxford OXI 3PJ, U.K
}

Received 26 February 1998; in revised form 3 June 1998

\begin{abstract}
Axisymmetric indentation of a flat surface is considered: specifically, the case of flat-ended indenter with rounded edges, and the case of a shallow cone with a rounded tip. Analytical solutions are obtained for the
normal and sequential tangential loading, in approximation), and for the complete interior stress for partial slip conditions (with the Cattaneo-Mindlin

Implications for strength of the contact are stress field in all these conditions.

the intention to shed more light in particular to the understanding refence to metallic or brittle materials, with tation' testings with nominally flat or conical indenters. It is found of common 'fretting fatigue' or 'indennominally zero for perfectly sharp flat or conical indenters, is well the strength of the contact, which is curvature. This is particularly true for the flat indenter, for, is well defined even for a small radius of than for the classical Hertzian indenter for a wide range, for which the strength is even significantly higher very attractive for design purposes. (C) 1999 Elsevier Science Letrical and loading conditions, rendering it Keywords: Contact stresses;
\end{abstract}

(ywords: Contact stresses; Flat punch; Conical punch; Indentation testing; Fretting fatigue testing

\section{Introduction}

Indentation testing of brittle or semi-brittle materials, such as ceramics or glasses, provides the potential to measure several important physical properties, from the fracture toughness to details of the test carried out, and the method stress states, depending on what is assumed, the blunt indenters, normally exemplified by the of analysis. Tests may be carried out either using typically a Vickers pyramid, a Berkovtich pyramid, cone crack test, and by sharp indenters, the important property that they provoke a lim, or a cone. The second class of indenters have

\footnotetext{
*Fax: 00441865279802
}

$0020-7683 / 99 / \$$ - see front matter 101999 Elsevier Science Ltd. All rights reserved
PII: S $0020-7683(98) 00186-3$ 
indenter, which produces a residual stress field promoting crack initiation, whilst the first have the virtue of producing a well defined non-singular stress field, although at the possible expense of causing crushing or plastic failure before a crack form. It should be noted that in his classic tests forming circular cracks, some of which were developed into 'cone frusta', Roesler in fact used a flat-ended indenter, rather than a sphere (Roesler, 1957). In this paper we wish to contribute to the literature quantifying the stress state developed for each of these classes of tests, by formulating solutions to:

(a) the problem of a flat-ended circular indenter having a finite curvature at the edge, rather than an abrupt edge. This permits us to use an elasticity solution to a half-space problem for both the indenter and the substrate, and so the usual idealization of a rigid indenter is not required.

(b) the problem of a conical indenter having a finite curvature at the tip. Again a half-space idealization for both the indenter and the indented material is used, so that, if the indenter is given a finite elasticity, the solution is appropriate only if the cone angle is large.

In addition to these examples of indenters used for testing, contacts involving nominally flat bearing surfaces occur widely in engineering practice, such as supporting feet/pads, or electrical brushes. There is therefore considerable practical motivation to study each of these geometries, and, in particular, to examine the influence of a round-off of either a cone apex or punch edge, as, in each case, the local singularity will be removed, and give rise to a well-posed problem within elasticity theory. Such rounding off will be present in practice, either because of limitations of the accuracy of the manufacturing process, or because localised plasticity during the first application of load will have caused local plastic flow, hence relieving the singularity.

Usually, the solution adopted for the flat (axisymmetrical) indenter is that due to Boussinesq (see, e.g. Timoshenko and Goodier, 1970) in which it is assumed that the indenter itself is rigid, and is pressed into a compliant, elastic, half-space. The contact is complete, the pressure distribution at the edges of the contact presents a square root singularity, and hence so are all the components of the local stress field $\left(\sigma_{i j} \sim 1 / \sqrt{ } r\right.$, where $r$ is the distance from the sharp corner). Physically, this is an unrealistic limiting case, because of the inevitability of plastic flow, and also because the assumption that the indenter is always much more rigid than the counter surface is not always tenable. ${ }^{1}$ Turning to the case of conical indenter, the half-space formulation is appropriate for shallow external angles, and gives a weak, logarithmic singularity at the tip (Sneddon, 1951), which again is, in practice, relieved by small scale plasticity. In both cases, the solutions are somewhat unsatisfactory, in that the strength of the contact is difficult to determine (concepts of Fracture Mechanics would be the best choice, although the singularity arising is not the classical one for a crack, and the implications of the stress intensity factor for the contact strength are unclear).

With these preliminaries, the present paper provides insight into the contact geometries described for normal indentation, full sliding, or partial slip of a static contact due to sequential application of normal and tangential loads; the last represents a generalisation of the celebrated Cattaneo-

\footnotetext{
'The proper elastic formulation for indenter and substrate of comparable elastic constants should be the one appropriate to a cylindrical domain, which requires an intense numerical effort, rendering it less attractive. Also, a singularity will still be present, although of lower degree than that implied by the Boussinesq solution (Khadem and
O'Connor, 1969).
} 


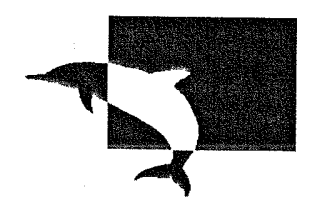

\section{University of Southampton}

To Accounts Payable

Rolls Royce plc

PO Box 31

Derby

DE24 8BJ
University of Southampton

Highfield

Southampton

SO17 1BJ

United Kingdom

Telephone 02380595000

Fax 02380592195
| Invoice No R $\quad 608801$

| 06-Jun-2005

$$
\begin{aligned}
& \mid \begin{array}{c}
\text { Account Code No } \\
\text { or Student (Reg No) } \\
\text { or Staff (Payroll No) }
\end{array} \\
& \mid \begin{array}{c}
\text { Customer Order No } \\
\text { Customer VAT No }
\end{array}
\end{aligned}
$$

Purchase Order No: 5000253553

SES No:1000117701

\section{DATUM Project}

Payment according to the above referenced purchase order

Miss H Angel

School Accountant

\begin{tabular}{|c|c|c|c|c|c|c|c|c|c|c|c|c|c|}
\hline$£$ & $\mathrm{P}$ & \multicolumn{10}{|c|}{ Code Number } \\
\hline 136,364 & 00 & $\mathrm{k}$ & $\mathrm{k}$ & 7 & 4 & $\mathrm{v}$ & 1 & 4 & $\mathrm{l}$ & 8 & 4 & 5 & 0 \\
\hline 23,863 & 70 & $\mathrm{x}$ & $\mathrm{b}$ & 6 & 2 & 9 & 1 & 1 & 0 & 9 & 4 & 1 & 5 \\
\hline & & & & & & & & & & & & & \\
\hline & & & & & & & & & & & & & \\
\hline
\end{tabular}

Sub-Total

VAT@ $17.5 \%$

Total

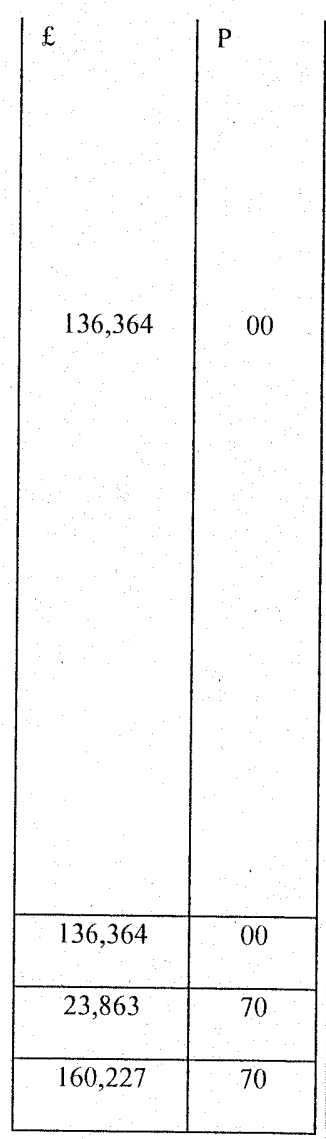

Payment terms strictly 30 days nett

University of Southampton VAT No GB 568630414

Please detach and return with your payment to:

Director of Finance, University of Southampton, Highfield, Southampton, SO17 1BJ

Account Code No.

Invoice No. R

608801

Name

Rolls Royce plc

Total $£$

$160,227.70$

Cheques should be made payable to 'University of Southampton'

Personal payments should be taken to the Chief Cashier, Finance Department, Room 2009, Administration Building. 

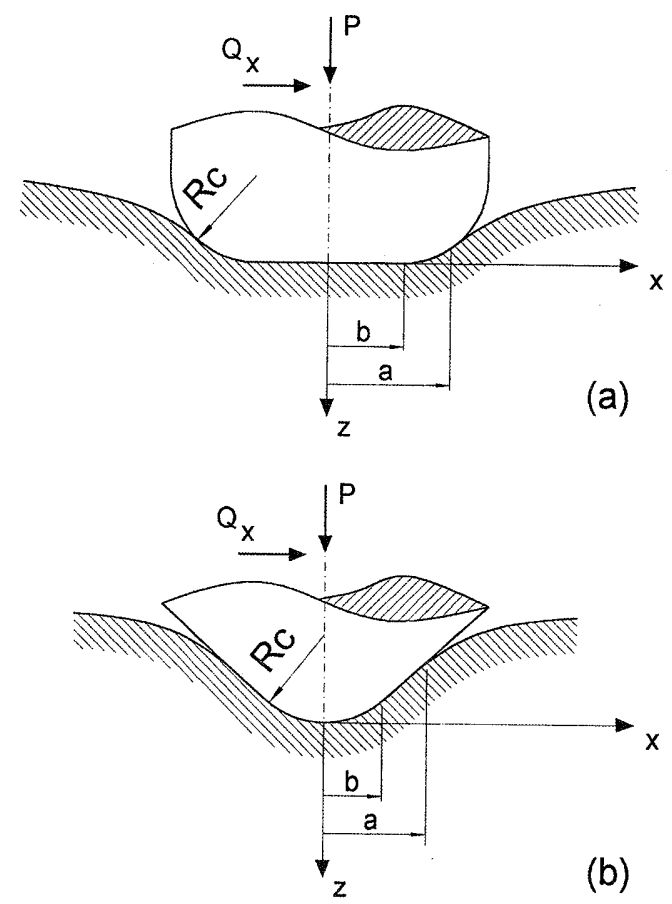
Fig. 1. Geometry and coordinate system of the problem. (a) Flat punch with rounded corner; (b) Conical punch with
rounded tip.

Mindlin problem (Cattaneo, 1938; Mindlin, 1949). Solutions are also developed for the interior stress field and issues related to strength of the contact addressed, with particular reference to elastic indentation testing.

\section{Formulation}

The problem will be formulated as an elastic punch indenting an elastic half-space, which may, in general, be of different elastic properties, although, if the bodies are not 'elastically similar' (see below), the contact must be lubricated for the solution to be precise, in order to eliminate the presence of shearing tractions. The geometries examined are shown in Fig. 1(a) and (b). The use of the half-space formulation is certainly justifiable for the rounded conical punch: it has been used successfully already for the sharp punch (Sneddon, 1951, using Hankel Transforms, and more recently, Sackfield et al. (1995), using Love's potentials and Abel integral equations), provided that the external cone angle, $\theta$, is small. In the case of flat punch, the half-space idealization will apply providing that the contact disk does not penetrate too far into the rounded off region, i.e. that, in the notation of Fig. 1(a), $a$ does not approach $b+R_{c}$, so that there is sufficient material around the contact to provide support. Analogous plane cases have been solved which show that, 
even if the radius of the edge curvature is only a small fraction of the contact disk radius, the solution is still quite satisfactory (Ciavarella et al., 1988a, b).

Well-established techniques for the solution of integral equations for elastic half-spaces can be used, as reported in the monographs on contact problems in the theory of elasticity (Shtaerman, 1949; Gladwell, 1980; Hills et al., 1993). In particular, for the normal axisymmetric contact problem general solutions, due to Shtaerman, are known, which we will follow here, with minor changes in notation; for tangential loading, a generalized version of the Cattaneo and Mindlin procedure (Cattaneo, 1938; Mindlin, 1949), due to the first author (Ciavarella, 1998a, b, c), will be used. Finally, the potential formulation will be employed for determining the complete interior stress field (see Love, 1927, Hills et al., 1993, Section 7.1).

\section{Normal loading}

We start with the governing integral equation for the general axisymmetric normal contact problem, which links the pressure distribution $p(r)$ over the contact area $S$, of radius $a$, to the relative surface normal displacements $u_{z}(r)$

$$
\begin{gathered}
A \\
2 \pi
\end{gathered} \iint_{S} p\left(r^{\prime}\right) \mathrm{d} S=u_{z}(r), \quad 0 \leqslant r \leqslant a
$$

where $r^{\prime}$ is the radial coordinate of the integration point, whose distance from the field point, of radial coordinate $r$, is given by $R$. Here $A$ is a measure of the 'composite compliance' of the bodies,
defined by

$$
{ }_{2}^{A}={ }_{E_{1}}^{1-v_{1}^{2}}+\begin{gathered}
1-v_{2}^{2} \\
E_{2}
\end{gathered}
$$

where $E_{i}$ is Young's modulus and $v_{i}$ Poisson's ratio of body $i$.

The equation written is precise only when no tangential radial tractions arise, which means that either: (i) the coefficient of interfacial friction, $f$, must be zero, or (ii) the materials are elastically similar, or, more precisely, Dundurs' second constant $\beta$ is zero, i.e.

$$
\begin{gathered}
1-2 v \\
\mu_{1}
\end{gathered}=\begin{gathered}
1-2 v_{2} \\
\mu_{2}
\end{gathered} .
$$

This removes the possibility of a change in the surface relative profile.

Side conditions give a unique solution of eqn (1), which are: (i) the surface normal displacements of the two bodies must match in the contact area; (ii) there can be no interpenetration external to the contact area; (iii) no tensile tractions can be transmitted between the bodies. The first two translate, respectively, to

$$
\begin{array}{ll}
u_{z}(r)=\alpha_{n}-z(r), & 0 \leqslant r \leqslant a \\
u_{z}(r)>\alpha_{n}-z(r), & a \leqslant r
\end{array}
$$


where $\alpha_{n}$ is the relative approach of two remote points, one in each body, and the indenter ${ }^{2}$ profile is described by $z(r)$. The solution, in the case when $p(a)$ is bounded (the so-called incomplete contact conditions), is (Shtaerman, 1949)

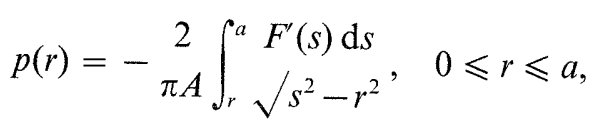

where the auxiliary function $F(r)$ has been introduced

$$
F(r)=\alpha_{n}-r \int_{0}^{r} \begin{gathered}
z^{\prime}(t) \mathrm{d} t \\
\sqrt{ } r^{2}-t^{2}
\end{gathered}, \quad 0 \leqslant r \leqslant a
$$

and $\alpha_{n}$ is given by (Shtaerman, 1949)

$$
\alpha_{n}=a \int_{0}^{a} z^{\prime}(t) \mathrm{d} t
$$

Equilibrium between the applied load and the pressure distribution can be written as (Shtaerman, 1949)

$$
P=\int_{0}^{a} 2 \pi r p(r) \mathrm{d} r={ }_{A}^{4} \int_{0}^{a} z^{\prime}(t) t^{2} \mathrm{~d} t
$$

The solution may be completed by the representation of stress and displacement fields in each body, but this point will be discussed in Section 5 .

\subsection{Flat punch with radiused corner}

In this case, the profile of the geometry is described by

$$
z(r)= \begin{cases}0, & 0 \leqslant r \leqslant b \\ k(r-b)^{2}, & b \leqslant r \leqslant a\end{cases}
$$

where $k$ is the curvature of the rounded part, i.e. $k=\left(1 / R_{c}\right)$, see Fig. 1(a). Let us start the solution from the load-contact radius relation. Computing the profile derivative from (10) and carrying out the integration of eqn (9), with the substitution $t=a \cos \varphi$, gives

$$
\int_{b}^{a} \sqrt{a^{2}-t^{2}}=\begin{gathered}
b^{3} \\
5 \cos ^{3} \varphi_{0}
\end{gathered}\left[3 \sin \varphi_{0}+\sin ^{3} \varphi_{0}-3 \varphi_{0} \cos \varphi_{0}\right]
$$

and we may obtain the result that the contact load $P$ is given by

\footnotetext{
${ }^{2}$ The case of two half-spaces having arbitrary profiles is readily incorporated by writing $z(r)=z_{1}(r)-z_{2}(r)$, where $z_{1}(r), z_{2}(r)$ describe the individual profiles.
} 


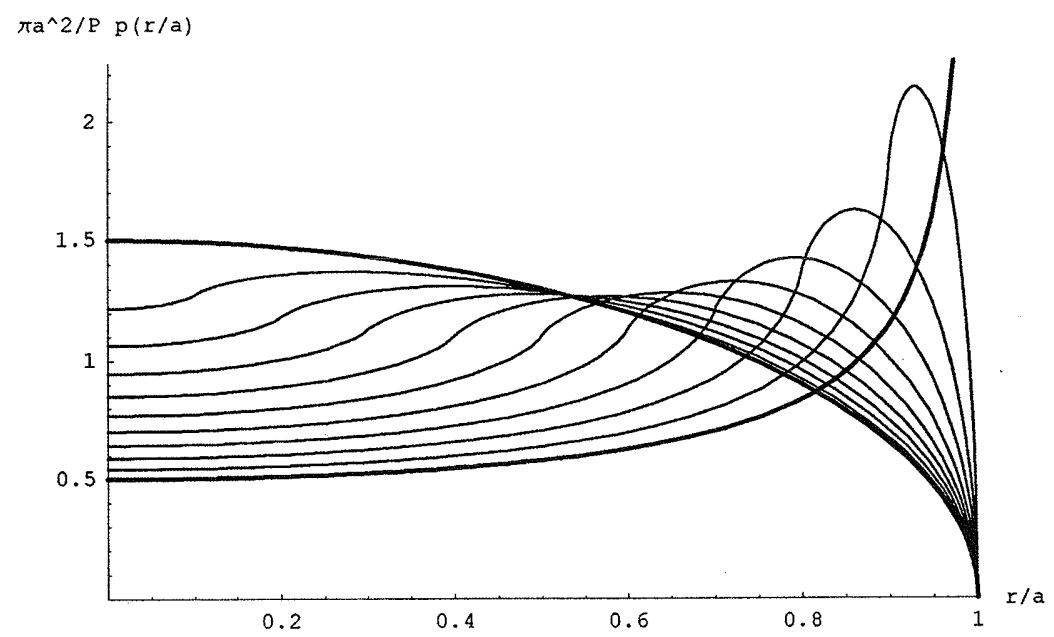

Fig. 2. Pressure distribution $\left(\pi a^{2} / P\right) p(r / a)$ for a flat indenter with rounded corners: $(b / a=0.0,0.1, \ldots, 1)$. Bold lines correspond to the limit cases: Hertzian $b / a=0$ and rigid sharp flat punch $b / a=1$.

$$
P=\begin{gathered}
2 k b^{3} 3 \sin \varphi_{0}+\sin ^{3} \varphi_{0}-3 \varphi_{0} \cos \varphi_{0} \\
3 A
\end{gathered} \cos ^{3} \varphi_{0},
$$

where the auxiliary angle $\varphi_{0}$ is introduced as

$$
\cos \varphi_{0}=\stackrel{b}{a} .
$$

The contact stiffness, i.e. the approach of remote points in the normal direction, $\alpha_{n}$, is obtained from (8) and (10) as

$$
\alpha_{n}=k a \int_{b}^{a}(t-b) \mathrm{d} t=k a \int_{0}^{\varphi_{0}}(a \cos \varphi-b) \mathrm{d} \varphi=k a\left(a \sin \varphi_{0}-b \varphi_{0}\right)
$$

and so

$$
\alpha_{n}=k b^{2} \begin{gathered}
\tan \varphi_{0}-\varphi_{0} \\
\cos \varphi_{0}
\end{gathered}
$$

Turning to the pressure distribution, this is now found directly by carrying out the integration of (6) as shown in Appendix I. The result, non-dimensionalized by the mean value $p_{m}=\left(P / \pi a^{2}\right)$, is

$$
\begin{gathered}
p(r) \\
p_{m}
\end{gathered}=\begin{gathered}
3 \cos \varphi_{0} \\
3 \sin \varphi_{0}+\sin ^{3} \varphi_{0}-3 \varphi_{0} \cos \varphi_{0}
\end{gathered} \Psi_{f l}\left(\begin{array}{l}
r \\
b
\end{array}\right), \quad 0 \leqslant r \leqslant a
$$

where the function $\Psi_{\mathrm{fl}}(r / b)$ is given in Appendix I. In particular, note that the pressure at the flat/round transition point (which is not the location of the maximum pressure, but is not far away) is obviously finite. The pressure distribution for several values of the ratio $b / a$ is plotted in Fig. 2. It may be appreciated that the transition from the Hertzian distribution $(b / a=0)$ towards 
the upper limit distribution of the rigid flat punch one $(b / a=1)$ is quite smooth, and gives a maximum pressure which is lower than the Hertzian when $b / a \leqslant 0.7$ (about). In this range, note that the pressure is quite close to uniform.

\subsection{Conical punch with rounded tip}

In the case of the conical punch, Fig. 1(b), the geometry defined by

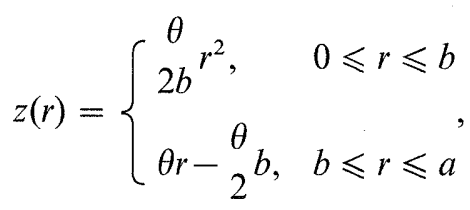

where $\theta$ is the external angle of the cone, that has to be small for the half-space approximation to be valid, i.e. $\theta \simeq \tan \theta$. Note that $\theta / b=k=1 / R_{\mathrm{c}}$.

Let us start, again, from the load-contact radius relation. Given the geometry eqn (17), and computing the derivative, we have from the integration of eqn (9), for $a>b$, the relation

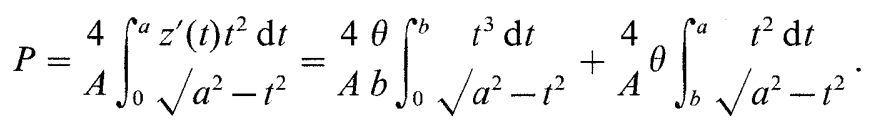

Now, with the substitutions $t=a \cos \varphi$, and $\cos \varphi_{0}=b / a$ we obtain

$$
\begin{array}{lc}
A P & b^{2} \\
4 \theta & 6 \cos ^{3} \varphi_{0}
\end{array}\left[4+3 \varphi_{0} \cos \varphi_{0}-3 \sin \varphi_{0}-\sin ^{3} \varphi_{0}\right] .
$$

Regarding the approach $\alpha_{n}$, we obtain, with the usual substitutions from (8)

$$
\alpha_{n}=\stackrel{b \theta}{\cos ^{2} \varphi_{0}}\left(1-\sin \varphi_{0}+\varphi_{0} \cos \varphi_{0}\right) .
$$

Finally, carrying out the integration of (6), as shown in Appendix II, the non-dimensionalized pressure is given by

$$
\underset{p_{m}}{p(r)}=\begin{gathered}
6 \cos \varphi_{0} \\
4+3 \varphi_{0} \cos \varphi_{0}-3 \sin \varphi_{0}-\sin ^{3} \varphi_{0}
\end{gathered} \Psi_{\operatorname{con}}\left(\begin{array}{l}
r \\
b
\end{array}\right), \quad 0 \leqslant r \leqslant a .
$$

Appendix II gives the explicit form of the function $\Psi_{\text {con }}(r / b)$. In the case $a<b$ the contact is obviously Hertzian, and so well-known relations apply (Shtaerman, 1949; Hills et al., 1993; Gladwell, 1980).

Figure 3 shows the pressure distribution for $b / a=0,0.1, \ldots, 1$. It may be appreciated that, as $b / a \rightarrow 0$ the maximum pressure (at the contact centre $r=0$ ) becomes increasingly high (indeed, it grows logarithmically). 


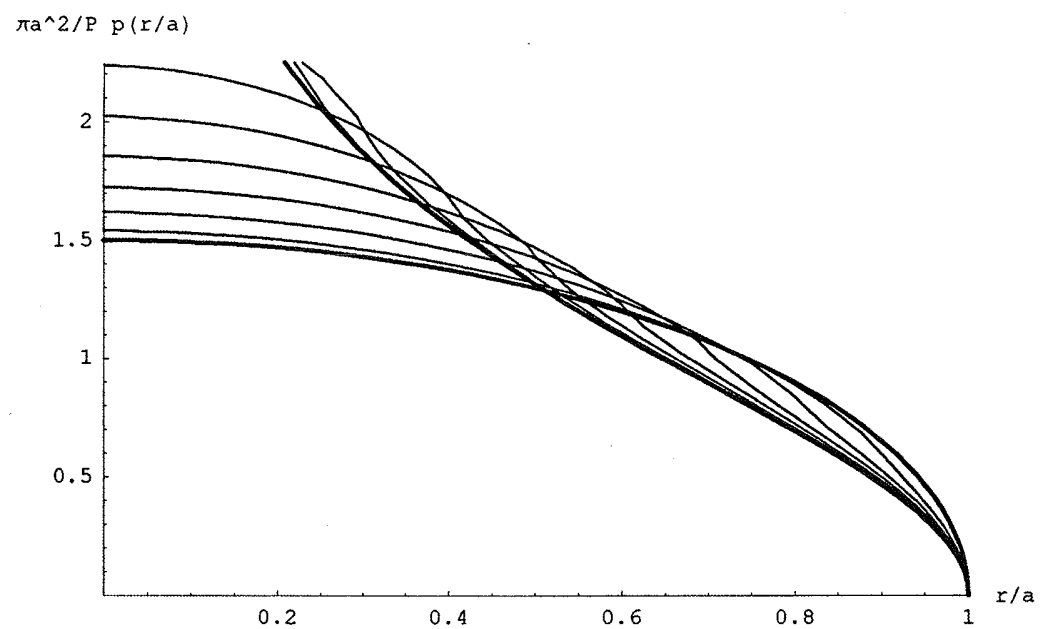

Fig. 3. Pressure distribution $\left(\pi a^{2} / P\right) p(r / a)$ for a conical indenter with rounded apex: $(b / a=0.0,0.1, \ldots, 0.9)$. Bold lines correspond to the limit cases: sharp conical punch $b / a=0$ and Hertzian $b / a=1$.

\subsection{Comparison}

Figure 4 shows a comparison between the two cases regarding the variation of load (for a given elastic compliance $A$ and geometry) with $b / a$. Note that the Hertzian value corresponds to $b / a=0$ and $b / a=1$, for the flat and conical case, respectively. As the quantities plotted are, $A P / k a^{3}$, $b A P / \theta a^{3}$, respectively, for flat and conical indenters, i.e. $A P R_{c} / a^{3}$ in both cases, the Hertzian limit case corresponds in respective limits.

Figure 5 shows the variation of approach in normal direction ( for a given load $P$ and compliance A) $\left(R_{c} \alpha_{n} / a^{2}\right)=\left(\alpha_{n} / k a^{2}\right)$ for flat (bold line) and $\left(R_{c} \alpha_{n} / a^{2}\right)=\left(b \alpha_{n} / \theta a^{2}\right)$ conical indenter as a function of $b / a$. Notice that the approach is always higher for the conical indenter, but since we are plotting the quantity $R_{c} \alpha_{n} / a^{2}$ for comparison purposes, this quantity goes to zero in the limit cases of sharp punch, as $R_{c}=0$. Notice that the variations of $R_{c} \alpha_{n} / a^{2}$ are not too far from linear with $b / a$.

\section{Tangential loading}

A solution will now be developed for the case of tangential loading, again assuming $\beta=0$ [i.e. eqn (3) holds], by appealing to a recent general result (Ciavarella, 1998a, b, for the plane problem, $1998 \mathrm{c}$, for 3-D problems). This solution, which will be briefly discussed below, is exact (i.e. it satisfies all contact requirements and Coulomb's friction law, including the normality rule), only (i) for plane problems or (ii) if the materials present no Poisson's effect, i.e.

$$
\gamma A={ }_{\mu_{1}}^{v_{1}}+{ }_{\mu_{2}}^{v_{2}}=0
$$

where $\gamma$ is now defined as the 'combined Poisson's ratio'. However, for a single axisymmetric 


\section{$\mathrm{APR}_{w} / a^{3}$}

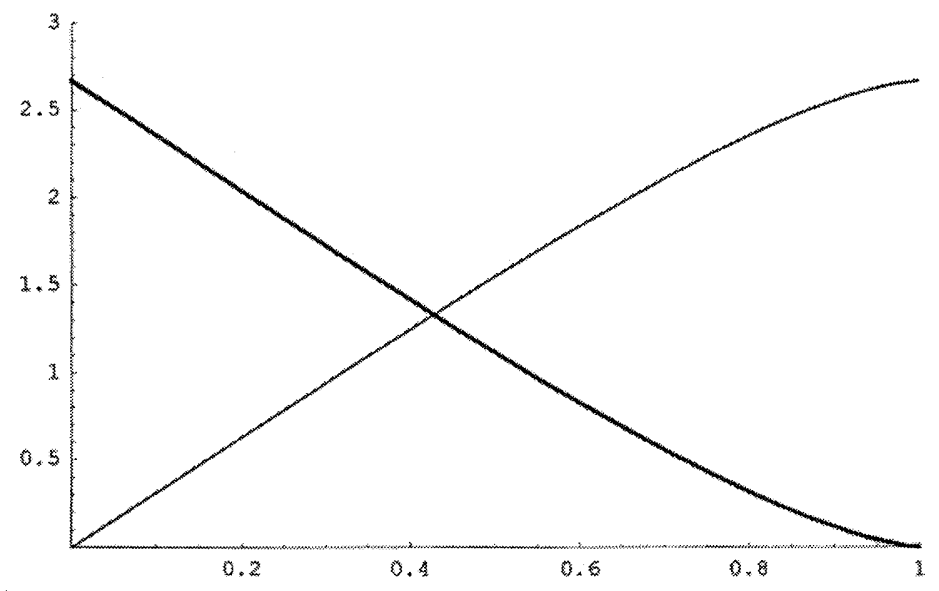

$b / a$

Fig. 4. Non-dimensional load $\left(A P R_{c} / a^{3}\right)=\left(A P / k a^{3}\right)$ for flat indenter (bold line) and $\left(A P R_{c} / a^{3}\right)=\left(b A P / \theta a^{3}\right)$ for a conical indenter as a function of $b / a$.

$$
\alpha \mathrm{R}_{\mathrm{c}} / \mathrm{a}^{2}
$$

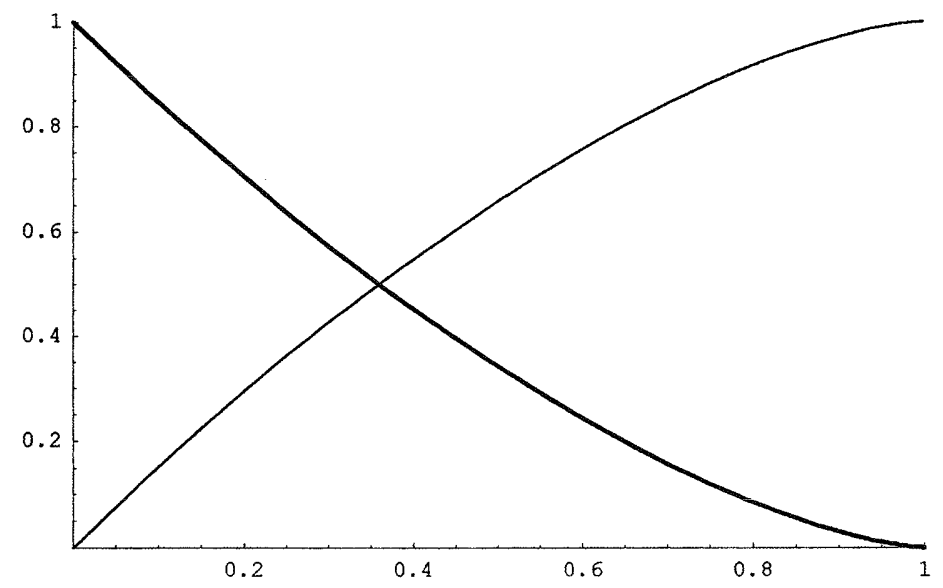

$\mathrm{b} / \mathrm{a}$

Fig. 5. Non-dimensional approach $\left(R_{c} \alpha_{n} / a^{2}\right)=\left(\alpha_{n} / k a^{2}\right)$ for flat indenter (bold line) and $\left(R_{c} \alpha_{n} / a^{2}\right)=\left(b \alpha / \theta a^{2}\right)$ for a conical indenter as a function of $b / a$. 
contact, the only approximation involved is the almost universally accepted one introduced by Cattaneo (1938) and Mindlin (1949), for the solution of the corresponding problem for Hertzian contacts. ${ }^{3}$ The exact solution implies a slightly non-circular stick zone, and requires a numerical non-axisymmetric solution, but the effort to do so is not justified by the small correction to the resultant stress state (Munisamy et al., 1994). ${ }^{4}$

Bearing these preliminaries in mind, consider the following loading sequence: apply first the normal force alone, so that the stick zone envelops the entire contact. A monotonically increasing shearing force, $Q_{x}$, will, therefore, give rise to a receding stick problem, according to Dundurs' classification (Dundurs, 1975), and we can therefore solve directly for any particular value of $Q_{x}$. A solution can be developed with the shearing traction distribution acting in the direction of the tangential force only, i.e. a distribution $q_{x}(x, y)$. Equilibrium requires

$$
Q_{x}=\iint_{S} q_{x}(x, y) \mathrm{d} x \mathrm{~d} y .
$$

Relative tangential displacement of surface particles within the stick zone must be constant, as Newton's law requires shearing tractions to be equal and opposite in the two bodies, so that

$$
u_{x}(x, y)=\delta_{x} ; \quad u_{y}(x, y)=\delta_{y}, \quad(x, y) \in S_{\text {stick }}
$$

where the rigid body displacement in the direction orthogonal to the load, $\delta_{y}$, is zero in the axisymmetric case, due to symmetry. Coulomb's law requires

$$
|\mathbf{q}(x, y)| \begin{cases}<f p(x, y), & (x, y) \in S_{\text {stick }} \\ =f p(x, y), & (x, y) \in S_{\text {slip }}\end{cases}
$$

and that shear traction $\mathbf{q}(x, y)=q_{x}(x, y) \mathbf{i}_{x}+q_{y}(x, y) \mathbf{i}_{y}$ must always oppose the direction of relative change in the direction of slip.

Let us therefore write the two integrals that relate the displacement of particles parallel with the surface to the surface tractions, together with the boundary condition (24), in the form

$$
\begin{aligned}
& \delta_{x}=(1+\gamma) U(x, y)-\gamma \frac{\partial^{2}}{\partial x^{2}} V(x, y), \quad(x, y) \in S_{\text {stick }} \\
& \delta_{y}=-\gamma \frac{\partial^{2}}{\partial x \partial y} V(x, y), \quad(x, y) \in S_{\text {stick }}
\end{aligned}
$$

where we define $U(x, y), V(x, y)$ as

\footnotetext{
${ }^{3}$ Although not recognized originally by the original authors (there is a footnote only in Mindlin (1953) and Dereciewicz's paper), the solution implies non-zero relative displacements $u_{y}$ in the slip area, so that the slip direction is not truly colinear with the shearing traction direction.

${ }^{4}$ For dissimilar elastic materials $(\beta \neq 0)$, the pressure distribution and shearing tractions in both the normal and, a fortiori and tangentially loaded problem will present a complicated pattern, and the stick-slip boundary a complicated shape, strongly dependent on the actual loading path.
} 


$$
U(x, y)=2 \pi \iint_{A} q_{x}\left(x^{\prime}, y^{\prime}\right) \mathrm{d} x^{\prime} \mathrm{d} y^{\prime}, \quad V(x, y)=2 \pi \iint_{S} R q_{x}\left(x^{\prime}, y^{\prime}\right) \mathrm{d} x^{\prime} \mathrm{d} y^{\prime}
$$

where $R=\sqrt{ }\left(x-x^{\prime}\right)^{2}+\left(y-y^{\prime}\right)^{2}$. From (27), follows that $V(x, y)$ has the form

$$
V(x, y)=g_{1}(x)+g_{2}(y)-{ }_{\gamma}^{\delta_{y}} x y, \quad(x, y) \in S_{\text {stick }} .
$$

Moreover, on considering $U(x, y)=\Delta V(x, y)$ as it follows from direct computation, where $\Delta$ is the 2-D Laplacian operator, (26) can be rewritten as

$$
\delta_{x}=g_{1}^{\prime \prime}(x)+(1+\gamma) g_{2}^{\prime \prime}(y), \quad(x, y) \in S_{\text {stick }}
$$

which is a differential equation with separate variables, with solution

$$
g_{1}^{\prime \prime}(x)=h_{1}, \quad g_{2}^{\prime \prime}(y)=h_{2}, \quad(x, y) \in S_{\text {stick }}
$$

satisfying the algebraic relation ${ }^{5}$

$$
\delta_{x}=h_{1}+(1+\gamma) h_{2} .
$$

Therefore, if we find a distribution $q_{x}$ satisfying the following condition in the stick area

$$
U(x, y)={ }_{A}^{2 \pi} \iint_{S} q_{x}\left(x^{\prime}, y^{\prime}\right) \mathrm{d} x^{\prime} \mathrm{d} y^{\prime}=\Delta V(x, y)=h_{1}+h_{2}, \quad(x, y) S_{\text {stick }}
$$

plus the other conditions in the slip area, and the obtained $V(x, y)$ is a second-degree polynomial in $x$ and $y$ in $S_{\text {stick }}$, this is an exact solution to the problem. This condition is always satisfied for axisymmetric problems ${ }^{6}$ like ours, and the solution of the problem can be obtained considering $q_{x}(r)=f p(r)-q_{x}^{*}(r)$ in the stick area, $q_{x}(r)=f p(r)$ in the slip area, and obtaining from eqn (33)using (1) for the contribution of the pressure - an integral equation in the stick area in terms of a corrective unknown part only

$$
2 \pi \iint_{S_{\text {stick }}} q_{x}^{*}\left(r^{\prime}\right) / f \mathrm{~d} S=u_{z}(r)-\begin{gathered}
h_{1}+h_{2} \\
f
\end{gathered}, \quad r \in S_{\text {stick }}
$$

${ }^{5} \mathrm{We}$ are assuming here the stick area is simply-connected.

${ }^{6}$ In fact, being $q_{x}$, and hence so $V$, function of $r$ only, eqn (33) reads

$$
\Delta V(r)=\begin{gathered}
\mathrm{d}^{2} V(r) \\
\mathrm{d} r^{2}
\end{gathered}+\begin{aligned}
& 1 \mathrm{~d} V(r) \\
& r \mathrm{~d} r
\end{aligned}=h_{1}+h_{2}
$$

has a general solution (neglecting logarithmic terms) $V(r)=\left[\left(h_{1}+h_{2}\right) / 4\right] r^{2}+c$.

More general cases of exact solutions are possible, as long as $V$ is a second-degree polynomial in $x$ and $y$. The most relevant are Hertzian elliptical contacts (as proved explicitly by Cattaneo, 1938) and plane problems (Ciavarella, 1997a, b). Indeed, for plane problems, with no dependence, say, on $y, \Delta V(x)=\left(\mathrm{d}^{2} V(x) / \mathrm{d} x^{2}\right)=h_{1}+h_{2}$ has a general solution $V(x)=\left[\left(h_{1}+h_{2}\right) / 2\right] x^{2}+c$. Other particular cases of such exact solutions may exist, with a more general shape of stick area, but the question of limited practical interest, as long as the surface profiles to produce that contact area and symmetry are of rather particular form, so that the property will hold only for very special values of geometry and load. 
i.e. the corrective part $q_{x}^{*}\left(r^{\prime}\right) / f$ can be found from a normal contact problem of the kind (1) for a reduced indentation. The details of the solution, including the proof that the inequalities in the slip area also translate to the non-interpretation condition of the corrective contact problem, are discussed in the cited papers, which provide further details. Here we just record that all the relevant quantities can be obtained from a superposition of the normal load solution, using the corrective load defined by

$$
Q_{x}^{*}=f P-Q_{x} .
$$

The only care required is in the calculation of displacements, but we find that in geometrically axisymmetric problems, with $h_{1}=h_{2}$ from eqn (32)

$$
\delta_{x}=\left(h_{1}+h_{2}\right)\left(1+\begin{array}{l}
\gamma \\
2
\end{array}\right)
$$

and therefore the approach in the tangential direction is related to the superposition of approach in the normal direction through the factor $[1+(\gamma / 2)]$, i.e.

$$
\alpha_{t}=\left(1+\begin{array}{l}
\gamma \\
2
\end{array}\right)\left(\alpha_{n}-\alpha_{n}^{*}\right)
$$

\subsection{Flat punch with radiused corner}

The size of the stick zone is given implicitly by the relation between $\phi_{0}$ and the corrective load $Q^{*}$, i.e. using (9) and (36), (12)

$$
\begin{gathered}
Q^{*} \\
f
\end{gathered}=\begin{gathered}
2 k b^{3} 3 \sin \phi_{0}+\sin ^{3} \phi_{0}-3 \phi_{0} \cos \phi_{0} \\
3 A
\end{gathered}
$$

where the auxiliary angle $\phi_{0}$, has been introduced as

$$
\cos \phi_{0}={ }_{c}^{b} .
$$

The approach of two remote points $\alpha_{t}$ in tangential direction is given by (38) and (15)

$$
\begin{gathered}
\alpha_{t} \\
f
\end{gathered}=k b^{2}\left(1+\begin{array}{l}
\gamma \\
2
\end{array}\right)\left(\begin{array}{c}
\tan \varphi_{0}-\varphi_{0} \\
\cos \varphi_{0}
\end{array}-\begin{array}{c}
\tan \phi_{0}-\phi_{0} \\
\cos \phi_{0}
\end{array}\right) .
$$

Lastly, the non-dimensional corrective shearing distribution is given by (35) and (16)

$$
\pi b^{2} Q_{x}^{*} q_{x}^{*}(r)=\begin{gathered}
3 \cos ^{3} \phi_{0} \\
3 \sin \phi_{0}+\sin ^{3} \phi_{0}-3 \phi_{0} \cos \phi_{0}
\end{gathered} \Psi_{f l}\left(\begin{array}{l}
r \\
b
\end{array}\right), \quad 0 \leqslant r \leqslant c
$$

where the function $\Psi_{f(}(r / b)$ is given in Appendix I. 


\subsection{Conical punch with rounded tip}

In the case of the conical punch, we need to distinguish two cases. First, for the case when $c>b$, so that the stick zone disk envelops the tip radius, the solution is given by the relations (9), (36) and (19), i.e.

$$
Q_{f}^{*}=\underset{3 A \cos ^{2} \phi_{0}}{2 \theta b^{2}}\left[4+3 \phi_{0} \cos \phi_{0}-3 \sin \phi_{0}-\sin ^{3} \phi_{0}\right]
$$

and the approach from (38) and (20)

$$
\alpha_{t}=b \theta\left(1+\begin{array}{l}
\gamma \\
2
\end{array}\right)\left(\begin{array}{cc}
1-\sin \varphi_{0}+\varphi_{0} \cos \varphi_{0} & 1-\sin \phi_{0}+\phi_{0} \cos \phi_{0} \\
\cos ^{2} \varphi_{0} & \cos ^{2} \phi_{0}
\end{array}\right)
$$

whereas the non-dimensionalized corrective shearing distribution is given by (35) and (21)

$$
Q_{x}^{*} q_{x}^{*}(r)=\begin{gathered}
6 \cos ^{3} \phi_{0} \\
4+3 \phi_{0} \cos \phi_{0}-3 \sin \phi_{0}-\sin _{0}^{3} \phi
\end{gathered} \Psi_{\text {con }}\left(\begin{array}{l}
r \\
b
\end{array}\right), \quad 0 \leqslant r \leqslant c .
$$

Appendix II gives again the function $\Psi_{\text {con }}(r / b)$.

In the case, $c<b$, when the stick zone lies within the radiused portion of the indenter, the nondimensionalized corrective shearing distribution is given by the Hertzian distribution (Hills et al., 1993)

$$
Q_{x}^{*} q_{x}^{*}(r)=\frac{3}{2} \sqrt{1-\left(\begin{array}{l}
r \\
c
\end{array}\right)^{2}, \quad 0 \leqslant r \leqslant c}
$$

and the relationship between the corrective shearing force, $Q^{*}$, and the size of the stick zone is given by

$$
Q_{f}^{*}=\begin{aligned}
& 8 \theta c^{3} \\
& 3 A b
\end{aligned}
$$

(where we have taken the curvature to be $\left.k=\left(1 / R_{c}\right)=\theta / b\right)$, and the approach of remote points in the tangential direction $\alpha_{t}$ is given by (38), (20) together with the Hertzian value (Hills et al., 1993)

$$
\alpha_{t}=b \theta\left(1+\begin{array}{l}
\gamma \\
2
\end{array}\right)\left(\begin{array}{cr}
1-\sin \varphi_{0}+\varphi_{0} \cos \varphi_{0} & c^{2} \\
\cos ^{2} \varphi_{0} & b^{2}
\end{array}\right)
$$

\section{Strength of the contact}

The explicit calculation of the interior stress field for a general profile may be obtained either by a series solution, using an expansion of the pressure in terms of Legendre polynomials, or by an analogous procedure expanding the contact profile itself, as described in, for example (Sackfield 
and Hills, 1988) or (Hills et al., 1993). However, a direct solution will be used here, which gives rise to a rapidly convergent numerical integration. ${ }^{7}$

\subsection{Theory}

The most suitable technique to deal with axisymmetric problems is to appeal directly to potential theory (see Love, 1927, for further details). It may be shown that the stress and displacement fields can be obtained by defining two harmonic potentials, $N$ and $T$, to model the effect of normal and tangential loading in $x$-direction, respectively. The displacement field is obtained as

$$
\begin{aligned}
& 2 \mu u_{x}=-(1-2 v) N_{x}-z N_{x z}+2 v T_{x x}+2 T_{z z}-z T_{x x z} \\
& 2 \mu u_{y}=-(1-2 v) N_{y}-z N_{y z}+2 v T_{x y}+2 T_{z z}-z T_{x y z} \\
& 2 \mu u_{z}=2(1-2 v) N_{z}-z N_{z z}+(1-2 v) T_{z x}-z T_{x z z}
\end{aligned}
$$

and the stress field as

$$
\begin{aligned}
& \sigma_{x x}=-N_{x x}-2 v N_{y y}-z N_{x x z}+2(1+v) T_{x z z}+2 v T_{x x x}-z T_{x x x z} \\
& \sigma_{y y}=-N_{y y}-2 v N_{x x}-z N_{y y z}+2 v T_{x x x}-z T_{x y y z} \\
& \sigma_{z z}=-N_{z z}-z N_{z z z}-z T_{x z z z} \\
& \sigma_{x y}=-(1-2 v) N_{x y}-z N_{x y z}+2 v T_{x x y}+T_{y z z}-z T_{x x y z} \\
& \sigma_{y z}=-z N_{y z z}-z T_{x y z z} \\
& \sigma_{z x}=-z N_{x z z}+T_{z z z}-z T_{x x z z} .
\end{aligned}
$$

The potentials are defined from

$$
\begin{aligned}
& N=\operatorname{Im}\left\{\int_{0}^{a} g(t)\left[z_{1} \ln \left(z_{1}+R_{1}\right)-R_{1}\right] \mathrm{d} t\right\} \\
& \left.T=\operatorname{Im}\left\{\int_{0}^{a} h(t)\left[\begin{array}{l}
1 \\
2
\end{array} z_{1}^{2}-\frac{1}{2} r^{2}\right] \ln \left(z_{1}+R_{1}\right)-\frac{3}{4} z_{1} R_{1}+\frac{1}{4} r^{2}\right] \mathrm{d} t\right\}
\end{aligned}
$$

where

$$
\begin{aligned}
& z_{1}=z+i t \\
& R_{1}^{2}=x^{2}+y^{2}+(z+i t)^{2}
\end{aligned}
$$

and, finally, $g(t)$ and $h(t)$ are real functions of $t$, obtainable in full sliding conditions as (Hills et al., 1993, f. 7.23)

\footnotetext{
${ }^{7}$ Except for the region of the surface contact disk, where numerical care is required for the nearly singular or singular integrals to be computed.
} 


$$
g(t)=\frac{h(t)}{f}=\frac{2 \mathrm{~d}}{\pi \mathrm{d} t} \int_{t}^{a} r p(r) \mathrm{d} r
$$

For our purposes, it is preferable to obtain the functions $g(t)$ and $h(t)$ in terms of the profiles, and to compare eqn (6.11) with eqn (7.23) of Hills et al. (1993), which gives, immediately

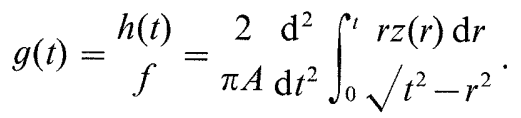

This is a more convenient form for our purposes, as the function $z(r)$ is elementary, whereas the function $p(r)$ is not. Elementary expressions will be obtained for $g(t)$ in both cases of a flat indenter with rounded corner and conical with rounded tip.

Notice that in the Cattaneo's partial slip regime, the required potential is $T_{p s}=T-T^{*}$, where $T$ is given by eqn (58), whereas $T^{*}$ is given by (58) by changing the upper limit of integration with $c$. Therefore, the 'partial slip potential' $T_{p s}$ can also be obtained by considering the integration in (58) extended from $c$ to $a$.

\subsection{Application to rounded indenters}

For the flat indenter, from (62) it is evident that $g(t)=0$ for $t<b$, whereas for $t>b$ the integral required is [using eqn (10)]

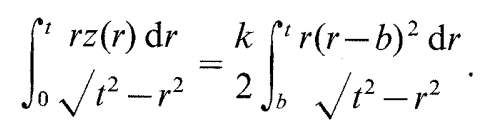

Therefore, we obtain, for full sliding, and $t>b$

$$
g(t)=\stackrel{h(t)}{f}={ }_{\pi A}^{2 k}\left[2\left(t^{2}-b^{2}\right)^{1 / 2}-b \arccos { }_{t}^{b}\right] .
$$

In the limit as $b \rightarrow 0$, we re-obtain the Hertzian value $g(t)=(h(t) / f)=(4 k / \pi A) t$.

For the conical indenter the relevant integral is given by (from 62) using (17)

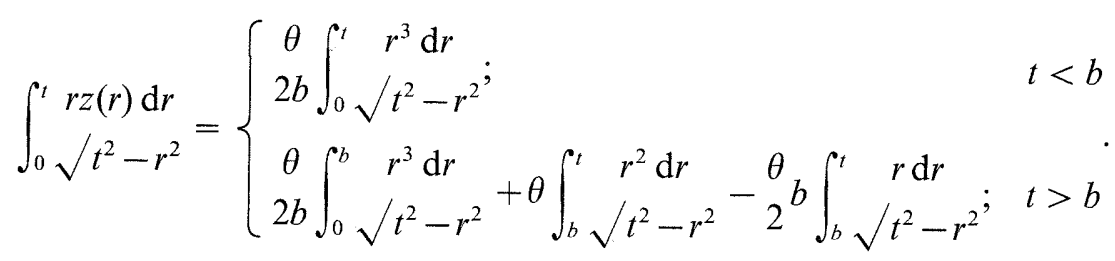

Now, with the help of some elementary integration, we get simply: in the case $t>b$

$$
g(t)={ }_{\pi A b}^{2 \theta}\left[2 t-2 \sqrt{ } t^{2}-b^{2}+b \arccos { }_{t}^{b}\right]
$$

whereas if $t<b$ 


$$
g(t)={ }_{\pi A b}^{4 \theta} t
$$

Note that in the limit as $b \rightarrow 0$, we re-obtain the conical punch value $g(t)=(h(t) / f)=(\theta / A)$. Also, in the limit as $b \rightarrow a$, we re-obtain the Hertzian value

$$
g(t)=\begin{array}{ll}
4 \theta & t \\
\pi A & a
\end{array}
$$

Once $g(t)$ is known, the determination of the stress field from eqns (51)-(53) or displacements from eqns (48)-(50) is relatively straightforward. Details of the computation of the potentials needed (57) and (58) and their derivatives are given in Sackfield and Hills (1988) and Hills et al. (1993), and reported briefly in Appendix III.

\subsection{Results}

It is impractical to present comprehensive results for the interior stress field, as there are many independent parameters involved, namely for each geometry, $(b / a, v)$ in the normal indentation case, $(b / a, v, f)$ for full sliding problems, and $(b / a, v, f, c / a)$ for partial slip problems; therefore, a complete study is impractical if we are not guided by some preliminary general considerations:

For normal loading

- where failure is controlled by yielding, there will be a transition between the two limiting cases of Hertzian indenter, where the maximum shear stress arises subsurface, and the sharp punches, where this maximum is at the singularity point. In the case of 'sharp' indenters softened in their intensity by the inclusion of a radius, there is a great deal of difference between the flat indenter and the cone, as in the latter case the strength is always lower than Hertzian, whereas for the flat ended punch the strength may be higher, as the pressure tends to be more 'uniform', over the contact patch.

- where failure is controlled by the initiation of surface defects and their propulsion, the maximum tension induced at the surface the most important quantity. However, as shown by Way (1941), the surface value outside the contact patch does not depend on the exact distribution of pressure, for purely normal loading, and therefore for very small defects the maximum tension is insensitive to the actual pressure distribution. Differences are found only for those cracks whose crack tip is sufficiently subsurface for the effect of the interior stress field to be of importance, but insufficiently deep for the zone of radial compression to be entered.

\section{For sliding problems}

- the maximum of tension always arises at the trailing edge of the contact patch, and its value for the conical punch, is therefore bound between the 'sharp' cone solution and the Hertzian, as lower and upper limits, respectively. Also, the maximum tension is strongly dependent on the value of the shearing force, but only more weakly controlled by the shearing traction distribution.

Guided by these general considerations, it is possible now to explore the strength in greater detail, splitting the discussion for the two geometries. 


\subsubsection{Flat indenter}

Normal loading. Figure 6(a)-(c) shows plots of the normalised von Mises yield parameter, $a^{2} \sqrt{ } J_{2} / P$, for representative cases of normal loading. As the ratio $(b / a)$ becomes very small the solution tends to the standard Hertzian case, whilst for very high $(b / a)$ the geometry tends to the flat-ended punch value. Perhaps surprisingly, varying $(b / a)$ causes the strength of the contact first to increase with respect to the Hertzian case, then to decrease very slowly. This can be seen in Fig. $6(a)$, which is for $b / a=0.25$, the maximum value of von Mises yield parameter, instead of lying at the subsurface point on the centreline as for the Hertzian case, moves to a region of almost constant value. In Fig. $6(\mathrm{~b})$, which is for $b / a=0.5$, the maximum lies in a wide, ring-shaped region close to the contact patch. Finally, in Fig. $6(\mathrm{c})$, when $b / a=0.75$, the maximum is again in a localized region, moving towards the contact edge, where the limiting sharp-cornered, flat case $b / a=1$ has its singularity.

Results from several plots of this kind are summarized in Fig. 7, which gives the elastic limit, $P / k a^{2}$, where $k$ is the yield strength in pure shear, for normal indentation as a function of the ratio $b / a$, according to von Mises' yield criterion: over the wide range $0<b / a<0.83$ the strength is in fact higher than the Hertzian case in the normal frictionless contact, ${ }^{8}$ with a maximum increase of more than $20 \%$, corresponding to values in the range $b / a=0.4-0.6$. This is because the pressure is close to uniform in that range (see Fig. 2), and the maximum of the yield parameter $a^{2} \sqrt{ } J_{2} / P$ moves from a well-defined point at the centreline to a region of nearly minimum value as described above: note that, in the 'optimal' uniform pressure case, for our choice of $v=0.3$, the optimal normalised von Mises' parameter is slightly greater than eight (Hills et al., 1993). For values of $b / a$ higher than 0.8 the strength decreases rapidly: therefore, for design purposes, it is safer to keep the value of $b / a$ (or, equivalently, the normalised load from eqn (12)), lower than, say, 0.6 .

Full slip. We turn now to the case where a tangential load, sufficient to cause sliding, has been applied. Figure 6(d)-(f) show representative cases of full sliding conditions. The results are summarized in Fig. 8 and show the dependence of the elastic limit $P /\left(a^{2} k\right)$ on the friction coefficient $f$. First, it is clear that, starting from the Hertzian configuration $b / a=0$, it is well known (Hills et al., 1993) that, for small coefficients of friction, less than about $f=0.33$ the severest state of stress remains below the surface. Above this value the maximum moves to the surface, showing a discontinuity in the plot, as the strength decreases much faster in the surface controlled region. The same behaviour is also exhibited by rounded flat contacts, although the transition subsurfacesurface tends to occur at lower coefficients of friction, and moves monotonically towards the limit of the sharp flat punch, where the severest state of stress is always on the surface, at the contact edge. From Fig. 8 several conclusions can be drawn: first, it appears that, for a high enough friction coefficient, the increase in strength of the contact over the Hertzian value, found in the range $b / a=0,0.83$ for the corresponding normal loading case ( $f=0$ in this figure) tends to decrease. In fact, for $b / a<0.83$, the strength of the contact becomes lower than the Hertzian case for surfacelimited cases. For higher values of $b / a$, the strength is always lower, as was expected. Therefore, it

\footnotetext{
${ }^{8}$ Notice that in the equivalent plane case a smaller range of $b / a$ values was found where this held $(0,0.55)$ (Ciavarella et al., 1997b). This is physically reasonable, as the maximum pressure close to the edges of the punch is lower than the analogous plane case, because of the axisymmetric conditions, as the pressure contributes to the load by a multiplicative factor of $2 \pi r$.
} 
(a)

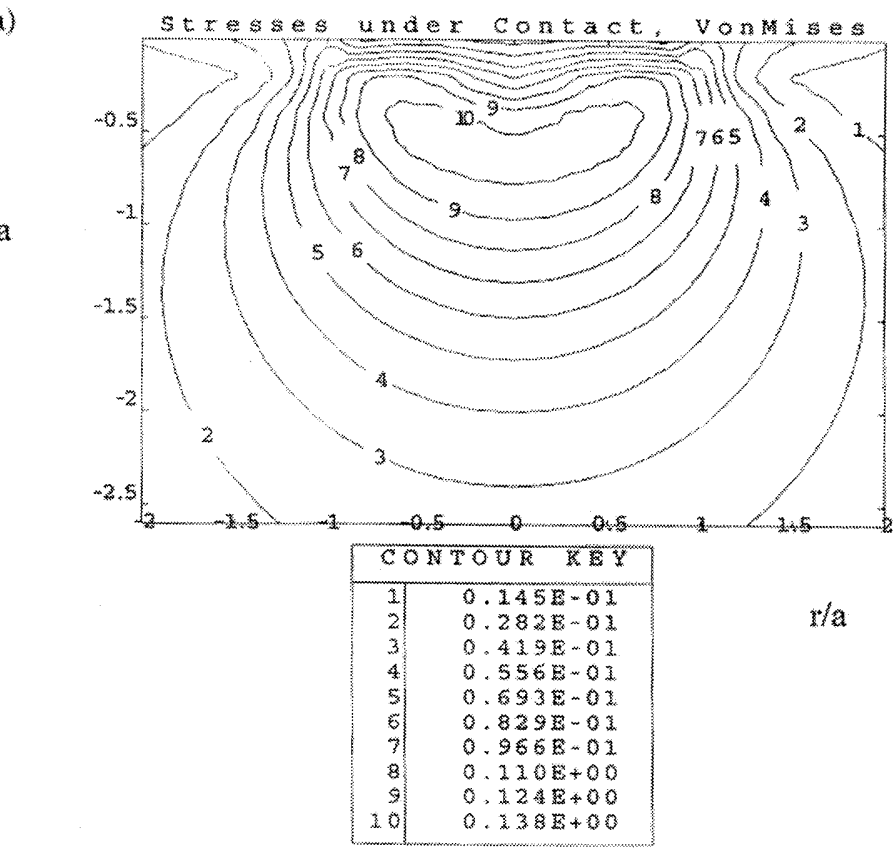

(b)

z/a

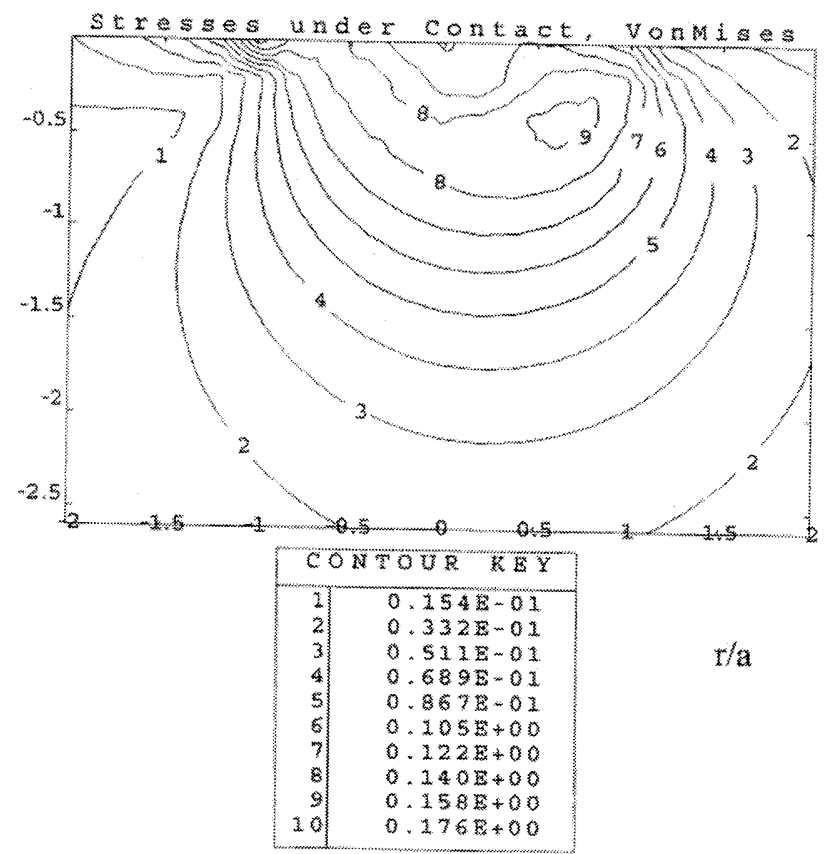

Fig. 6. Example contour plots of von Mises' yield parameter $a^{2} \sqrt{ } J_{2} / P$, for flat indenter under sliding conditions: (a) $b / a=0.25, f=0.0$; (b) $b / a=0.25, f=0.3$; (c) $b / a=0.5, f=0.0$; (d) $b / a=0.5, f=0.3$; (e) $b / a=0.75, f=0.0$; (f) $b / a=0.75, f=0.3(v=0.3)$. 
(a)

$z / a$
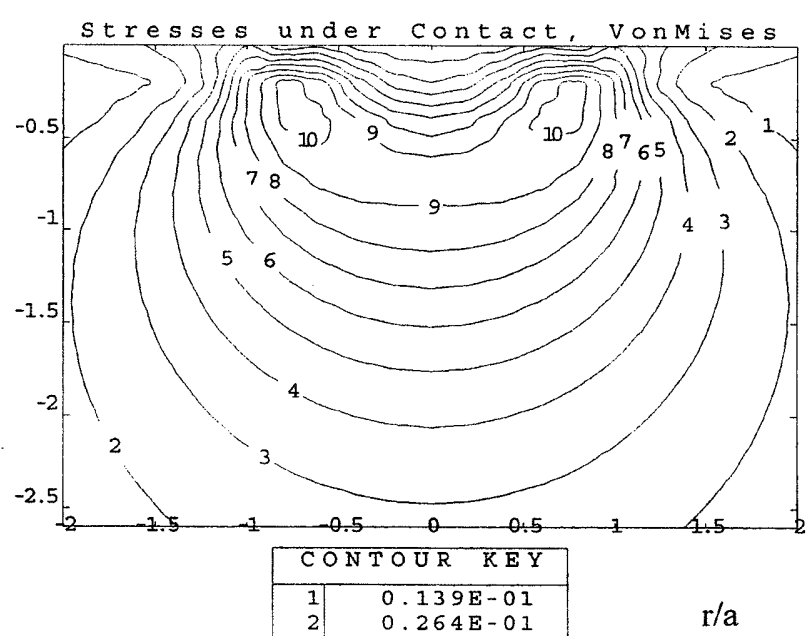

(b)

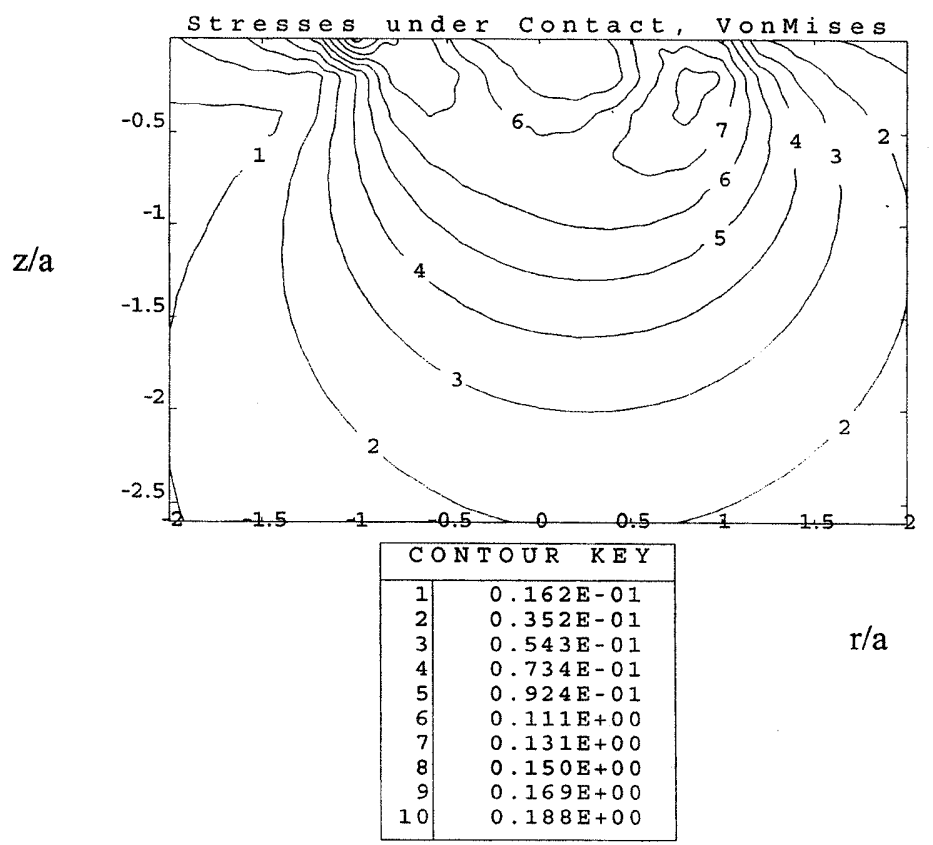

Fig. 6-continued. 


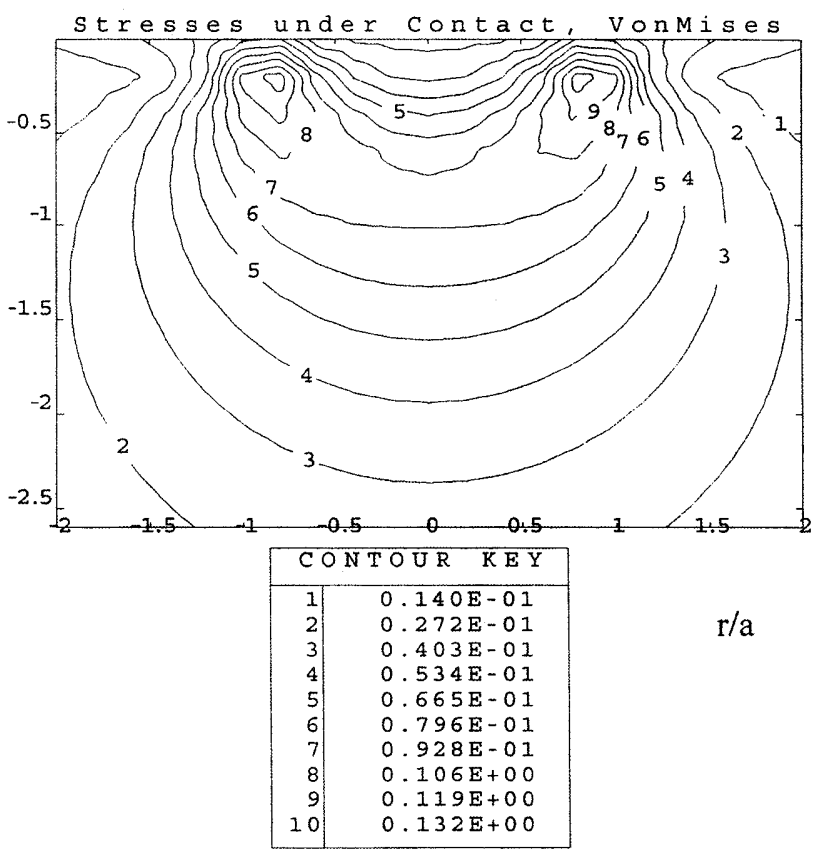

$\mathrm{z} / \mathrm{a}$

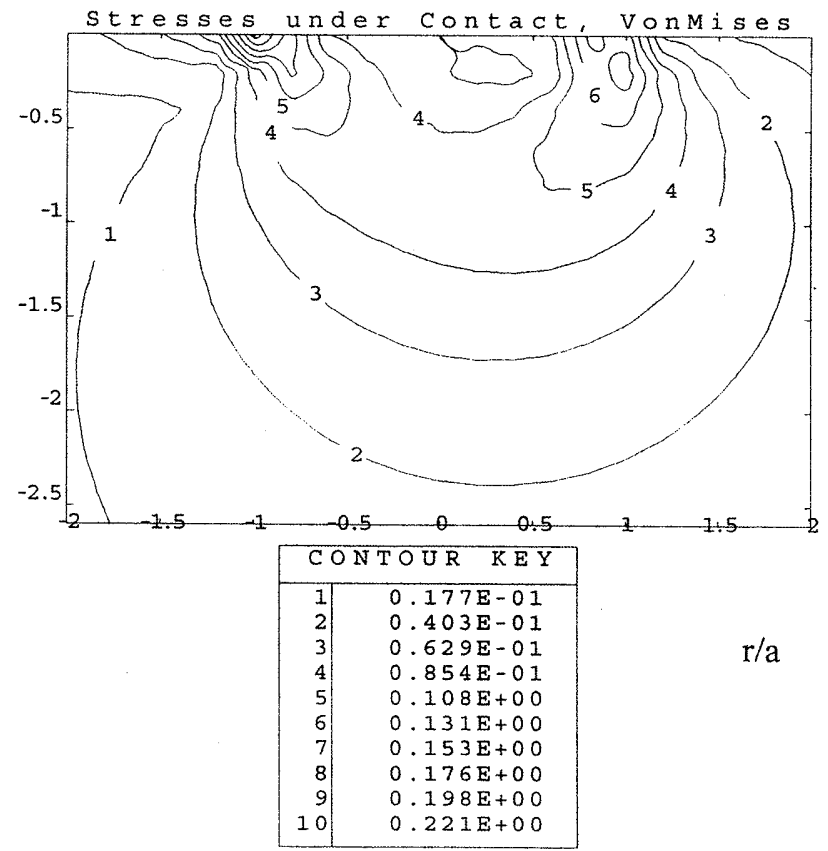

Fig. 6 -continued. 


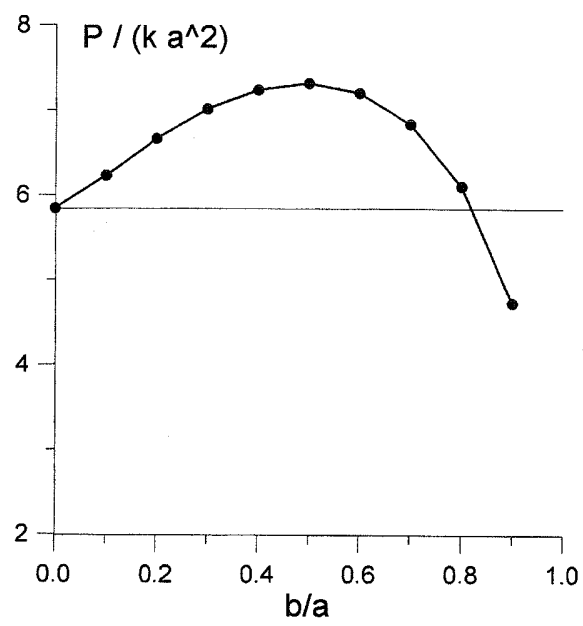

Fig. 7. Elastic limit $P / k a^{2}$, for normal indentation of a flat indenter, as a function of $b / a$. The horizontal line gives the strength for a Hertzian indenter $\left(P / k a^{2}\right) \cong 5.84$. The flat indenter has a higher strength for $b / a<0.83(v=0.3)$.

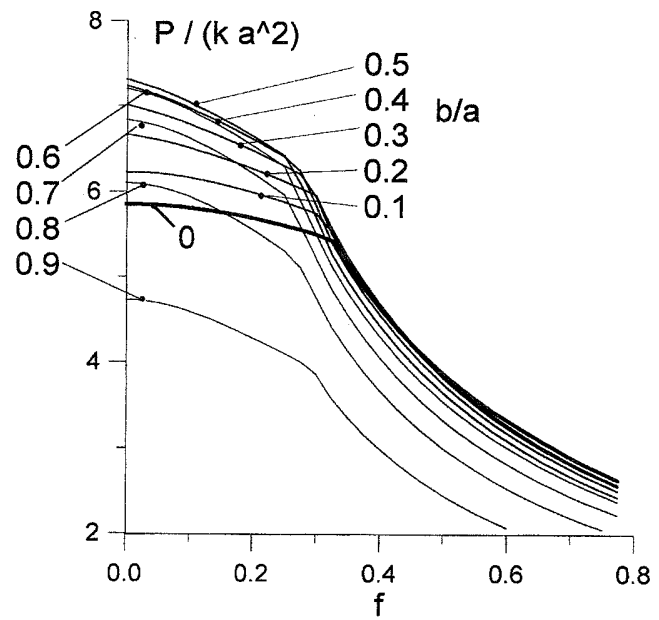

Fig. 8. Elastic limit $P / k a^{2}$, for sliding indentation of a flat indenter, as a function of the coefficient of friction, $f$. Ratio $b / a$ varying from $b / a=0$ (Hertzian indenter) to $b / a=0.9$ with steps $0.1(v=0.3)$.

can be said that there is a good deal or reason to keep the strength subsurface controlled, particularly if the contact is designed to take advantage of the rounded flat configuration with respect to the classical Hertzian one.

Turning to the maximum tension arising at the trailing edge of the contact, of relevance for the 


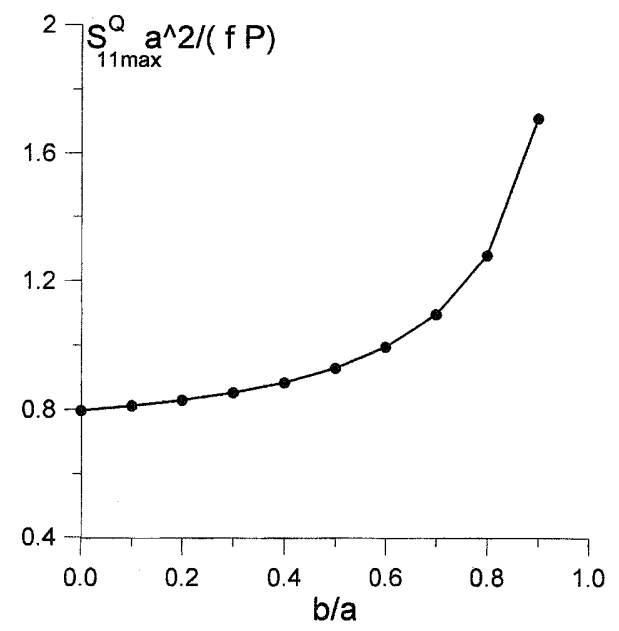

Fig. 9. Non-dimensional maximum tension $\left(a^{2} / f P\right) \sigma_{11}^{\max , Q}$ arising in the surface, trailing edge of the contact, for the case of a flat indenter under full sliding conditions, as a function of $b / a(v=0.3)$. Notice that the tension due to the normal load, which is independent on $b / a$, can be readily added and is $\left(a^{2} / P\right) \sigma_{11}^{\max , P}=0.0637$. For $b / a \rightarrow 1$ the maximum tension tends to infinity.

initiation and propulsion of surface cracks, it should be noted, from Fig. 9, that the maximum increases steadily with $b / a$, although the increase becomes particularly steep for $b / a$ higher than $\approx 0.8$. This region must be examined carefully, if the contact lies in this region and crack propagation is a possibility.

\subsubsection{Conical indenter}

The analysis just given for the flat indenter is here repeated for the cone. It is clear, however, that there is no advantage over a Hertzian design, apart from a minor improvement, under some conditions, in the maximum tension. Figure 10(a)-(c) shows plots of the normalised von Mises yield parameter, $a^{2} \sqrt{ } J_{2} / P$, for representative cases of normal loading. The concentration of tension occurs in a point subsurface on the centreline of the contact, which moves from the centre for the sharp cone case $b / a=0$, to the Hertzian value for $b / a=1$. This appears more clearly in Fig. 11, which shows plots of the normalised elastic limit $P /\left(a^{2} k\right)$, where $k$ is the yield strength in pure shear, as a function of the ratio $b / a$. It may be seen that the strength here is always lower compared with the Hertz limiting case, as expected, since the pressure distribution here is more localized near the centre of the contact. As the elastic limit in the sharp wedge case is theoretically zero, the major result of the present investigation is to show that for a ratio $b / a$ as small as 0.1 the elastic limit is still about half the value for the Hertzian case.

Full slip. Figure 10(d)-(f) show plots of $a^{2} \sqrt{ } J_{2} / P$, for representative cases of tangential loading. The results are summarized in Fig. 12, where the dependence of the elastic limit $P\left(a^{2} k\right)$ on the friction coefficient $f$ is given. It may be noted that the point where the strength becomes surface controlled is at a value of $f$ which decrease with $b / a$. This is consistent with the observation that, 

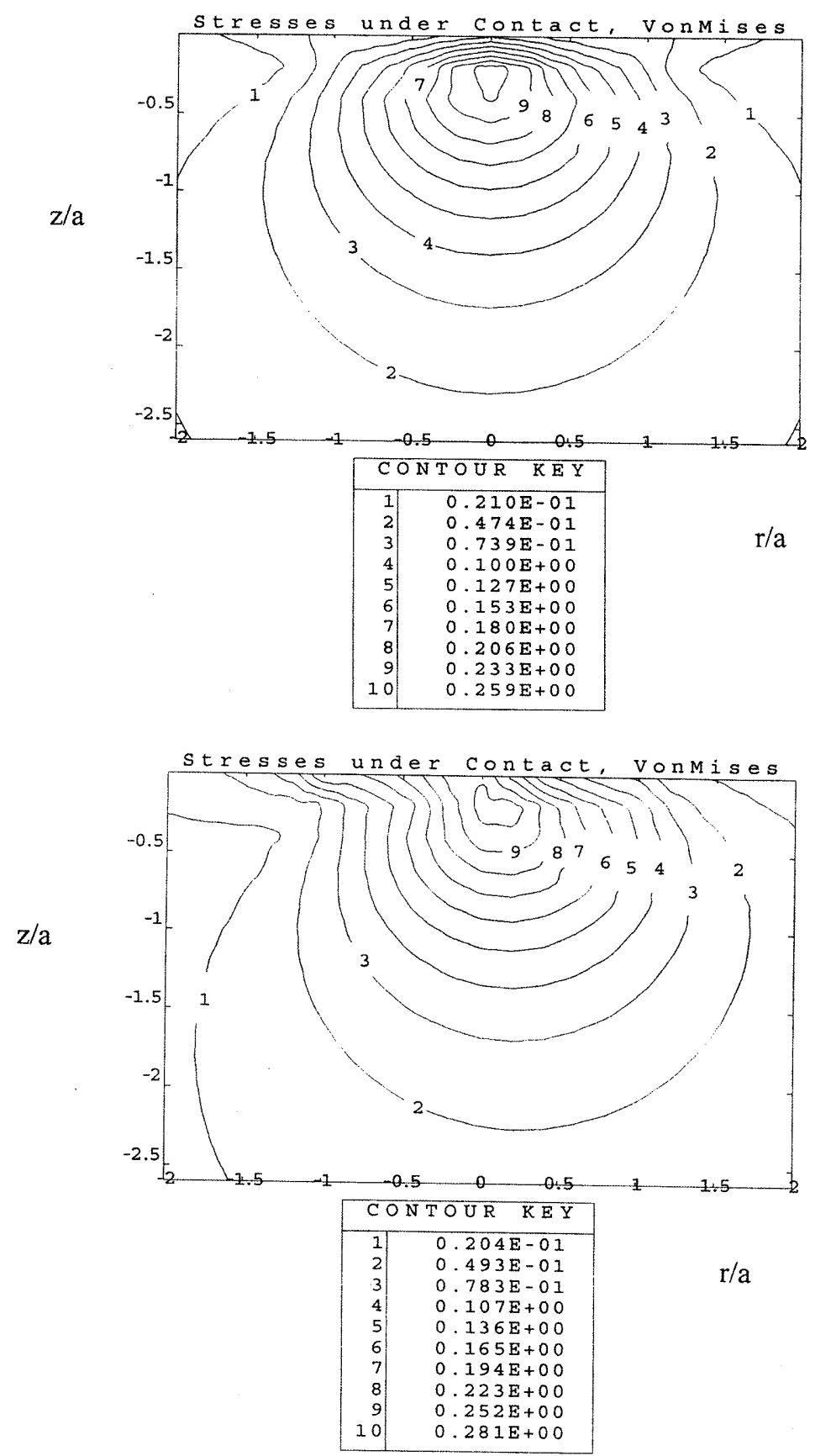

Fig. 10. Example contour plots of von Mises' yield parameters $a^{2} \sqrt{ } J_{2} / P$, for conical indenter under sliding conditions: (a) $b / a=0.25, f=0.0$; (b) $b / a=0.25, f=0.3$; (c) $b / a=0.5, f=0.0$; (d) $b / a=0.5, f=0.3$; (e) $b / a=0.75, f=0.0$; (f) $b / a=0.75, f=0.3(v=0.3)$. 


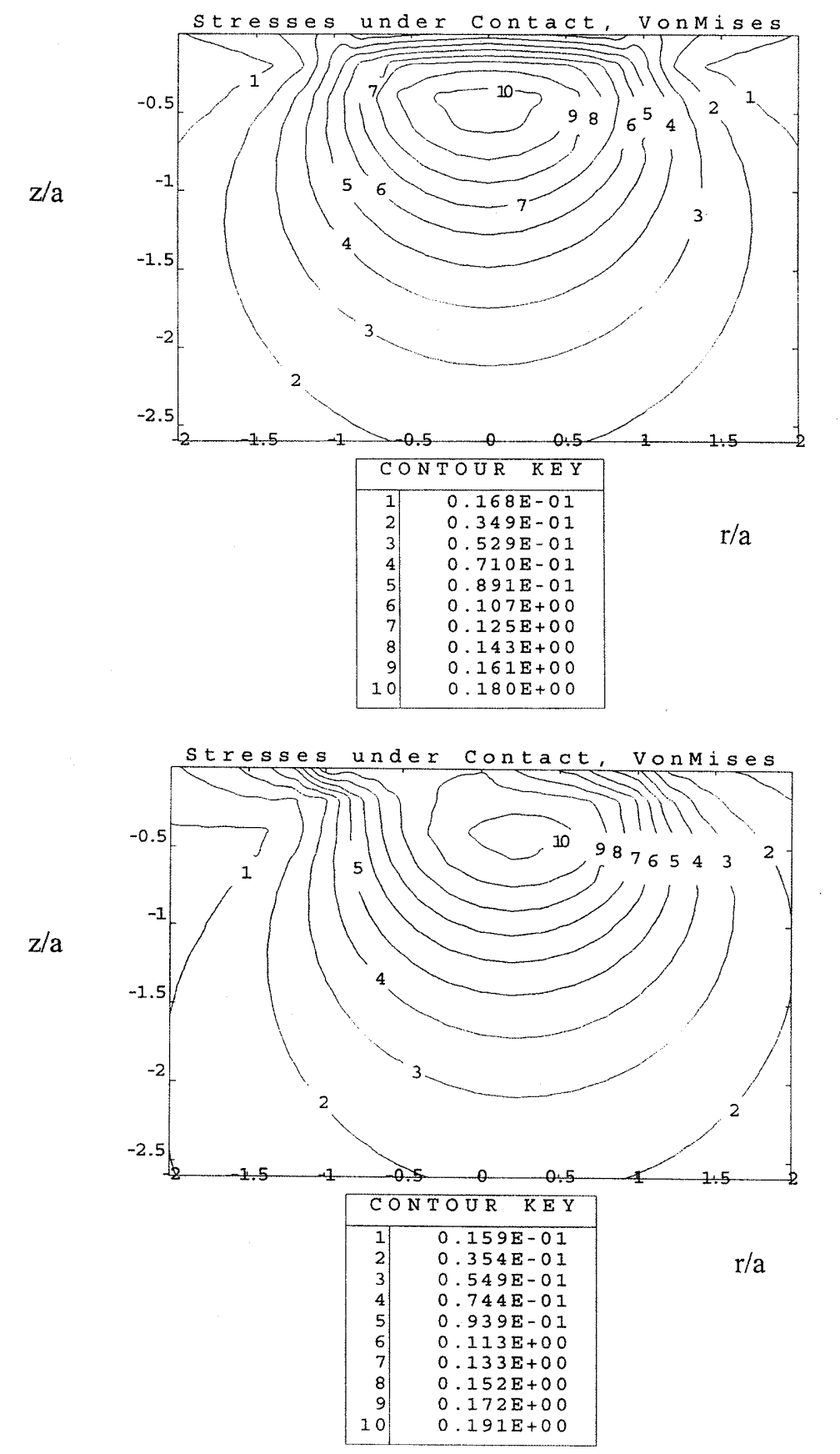

Fig. 10-continued. 


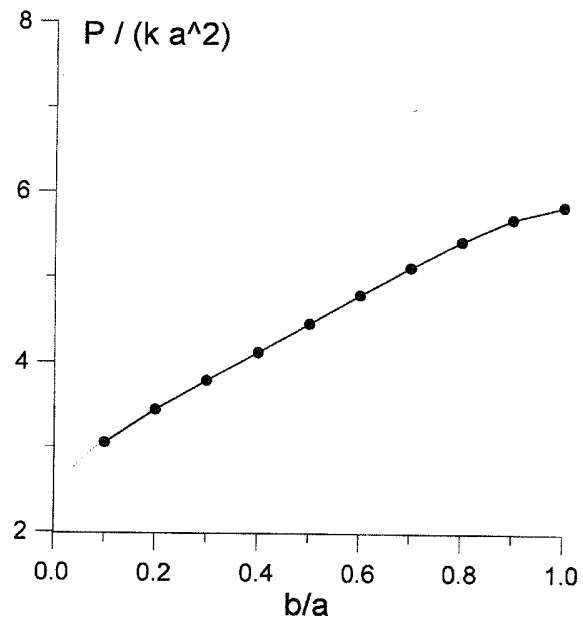

Fig. 11. Elastic limit $P / k a^{2}$, for normal indentation of a conical indenter, as a function of $b / a$. The upper limit for $b / a=1$ gives the strength for a Hertzian indenter $\left(P / k a^{2}\right) \cong 5.84(v=0.3)$.

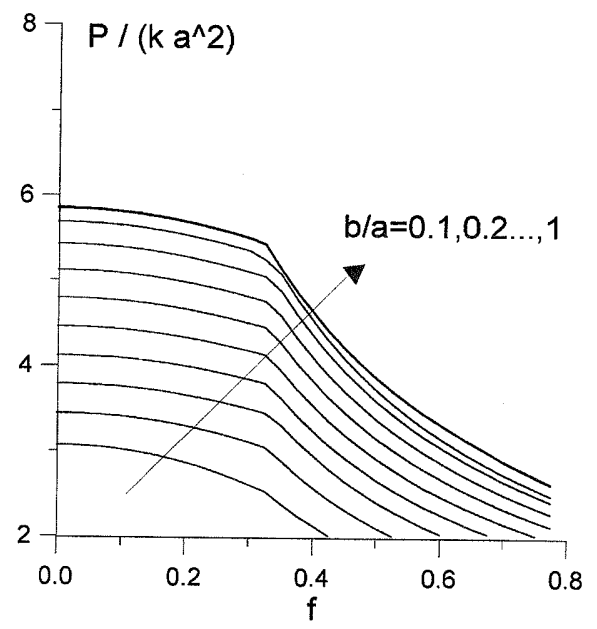

Fig. 12. Elastic limit $P / k a^{2}$, for sliding indentation of a conical indenter, as a function of the coefficient of friction, $f$. Ratio $b / a$ varying from $b / a=0.1$ to $b / a=1$ (Hertzian indenter) with steps $0.1(v=0.3$ ).

for the limiting sharp-point case, the strength, which is zero, is always controlled by the singular point in the centre of the contact surface.

Also of importance is the maximum tension induced at the surface, as this is the quantity which is responsible for initiating surface defects and their initial propulsion as cracks. The transition from the 'sharp' solution to the Hertzian is displayed in Fig. 13. The variation is not particularly enlightening. 
case, but the transition to the infinitely higher concentration predicted by the sharp flat punch can be considered slow.

For design purposes, a complete set of practical diagrams has been given, for normal or full sliding loading conditions. In the partial slip regime, since the corrective shear distribution always reduces the local value of the shearing traction, and since they are the principal quantities controlling yield, the elastic limit for the full sliding can be considered an upper bound for strength, therefore allowing a safe design.

\section{Appendix I: Pressure distributions for the flat punch}

In order to obtain the pressure distribution, according to the procedure given in Section 3, let us calculate the auxiliary function $F(r)$, defined in eqn (7), so that, considering the profile in eqn (10)

$$
F(r)=\left\{\begin{array}{ll}
\alpha_{n}, & 0<r<b, \\
\alpha_{n}-k r \int_{b}^{r}(t-b) \mathrm{d} t r^{2}-t^{2} & b<r \leqslant a
\end{array} .\right.
$$

Now, putting $t=r \cos \theta$, we obtain

$$
\int_{b \sqrt{ } r^{2}-t^{2}}^{r}=\sqrt{ } r^{2}-b^{2}-b \arccos \frac{b}{r}
$$

so that the derivative of $F(r)$ simplifies to

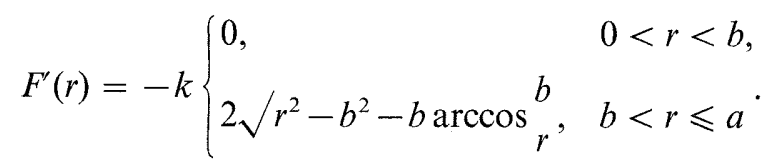

Hence the pressure can be obtained from eqn (6), as

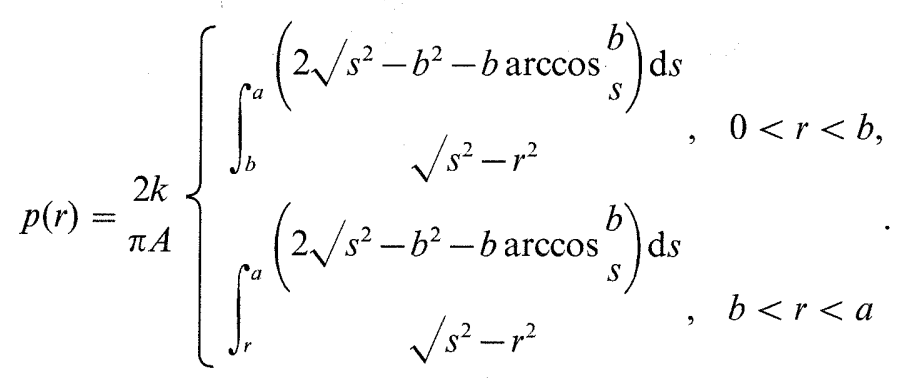

Assume $s=b / \cos \varphi$, so that $a=b / \cos \varphi_{0}, \mathrm{~d} s=b \tan \varphi / \cos \varphi \mathrm{d} \varphi$, and introduce the function $\Psi_{f(}(x)$ as 


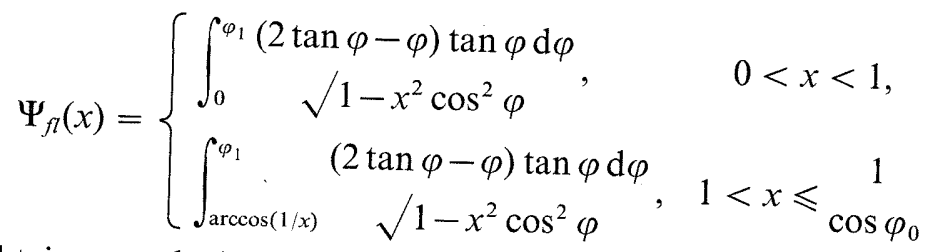

to obtain, on substituting the value of the load, the final expression, eqn (16).

\section{Appendix II: Pressure distribution for the conical punch}

Finally, to obtain the pressure distribution, let us calculate the auxiliary function $F(r)$, from $(7)$
r the profile (17)

$$
F(r)=\left\{\begin{array}{cl}
\alpha_{n}-r_{b}^{2}, & 0<r<b, \\
\alpha_{n}-r_{b}^{\theta}\left(r-\sqrt{ } r^{2}-b^{2}\right)-r \theta \arccos b / r, & b<r \leqslant a
\end{array}\right.
$$

so that the derivative of $F(r)$ simplifies to

$$
-F^{\prime}(r)=\theta\left\{\begin{array}{ll}
2 r / b, & 0<r<b, \\
2 r / b-2 / b \sqrt{ } r^{2}-b^{2}+\operatorname{arcos}{ }_{r}, & b<r \leqslant a
\end{array} .\right.
$$

Therefore, the pressure can be obtained from eqn (6), as

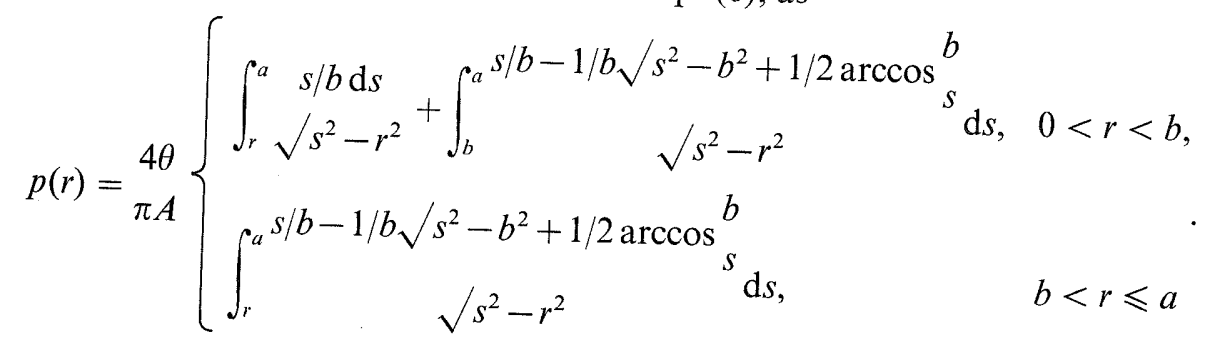

Apart from the first integral (where we use $s=b \cos \varphi$ ), we put $s=b / \cos \varphi$, so that $a=b / \cos \varphi_{0}$, $\mathrm{d} s=b \tan \varphi / \cos \varphi \mathrm{d} \varphi$, and use the variable $x=r / b$. Introducing now the function $\Psi_{\text {con }}(x)$ as

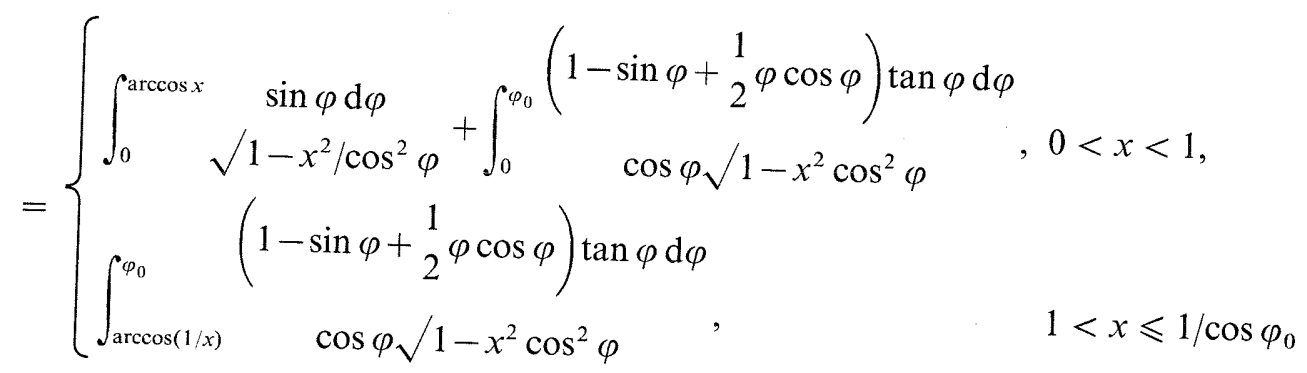

we obtain, considering the load eqn (19), eqn (21). 


$$
q^{4}=[z+\rho \cos (\alpha / 2)]^{2}+[t+\rho \sin (\alpha / 2)]^{2}
$$

and finally

$$
\begin{aligned}
& \tan \alpha=\begin{array}{c}
2 z t \\
r^{2}+z^{2}-t^{2}
\end{array}, \quad 0 \leqslant \alpha \leqslant \pi \\
& \tan \beta=\begin{array}{l}
t+\rho \sin (\alpha / 2) \\
z+\rho \cos (\alpha / 2)
\end{array}, \quad 0 \leqslant \beta \leqslant \pi .
\end{aligned}
$$

\section{Acknowledgements}

The author is pleased to acknowledge the support from CNR-Consiglio Nazionale delle Ricerche (Borsa per l'estero 203.07.26 del 12.9.96), for his visit to Oxford University, permitting also the completion of the present work. He is indebted to Dr D. A. Hills of the University of Oxford and Lincoln College, Oxford, U.K., for his suggestions.

\section{References}

Cattaneo, C., 1938. Sul contatto di due corpi elastici: distribuzione locale degli sforzi. Rendiconti dell'Accademia Nazionale dei Lincei 27, 342-348, 434-436, 474-478.

Ciavarella, M., 1998a. The generalized Cattaneo partial slip plane contact problem. I-Theory. Int. J. Solids Struct. 35 (18), 2349-2362.

Ciavarella, M., 1998b. The generalized Cattaneo partial slip plane contact problem. II-Examples. Int. J. Solids Struct. 35 (18), 2363-2378.

Ciavarella, M., 1998c. Tangential loading of general 3-D contacts. J. of Appl. Mech.-ASME, accepted.

Ciavarella, M., Hills, D.A., Monno, G., 1998a. Contact problems for a wedge with rounded apex. Int. J. Mech. Sci., accepted.

Ciavarella, M., Hills, D.A., Monno, G., 1998b. Influence of rounded corners on indentation by flat punches. IMechE J. of Mech. Eng. Sci., accepted.

Dundurs, J., 1975. Properties of elastic bodies in contact. In: de Pater, A.D., Kalker, J.J. (Eds.), The Mechanics of the Contact between Deformable Bodies. Delft University Press, pp. 54-66.

Gladwell, G.M.L., 1980. Contact Problems in the Classical Theory of Elasticity. Sitjhoff and Nordhoff, Alphen aan Olen Rijn.

Khadem, R., O'Connor, J.J., 1969. Adhesive or frictionless compression of an elastic rectangle between two identical elastic half-spaces. Int. J. Eng. Sci. 7, 153-168.

Hills, D.A., Nowell, D., Sackfield, A., 1993. Mechanics of Elastic Contacts. Buttermann-Heinemann, Oxford.

Mindlin, R.D., 1949. Compliance of elastic bodies in contact. J. Appl. Mech. 16, 259-268.

Roesler, F.C., 1957. Brittle fracture near equilibrium. Proc. Phys. Soc. 69, 981-992.

Sackfield, A., Hills, D.A., 1988. Sliding of a general axi-symmetric point contact. J. Tribology, 110, 492-498.

Sackfield, A., Truman, C.E., Hills, D.A., 1995. Contact Mechanics of wedge and cone indenters. Int. J. Mech. Sci. 37 (3), 261-275. 
Shtaerman, I. Ya., 1949. Contact Problem of the Theory of Elasticity. Moscow, Leningrad, Gostekhteoretizdat. Available from the British Library in an English translation by Foreign Technology Div., FTD-MT-24-61-70, 1970. Sneddon, I.N., 1951. Fourier Transforms. McGraw-Hill, New York.

Neddon, I.N., 1963. The relation between load and penetration in the axisymmetric Boussinesq problem for a punch of arbitrary profile. Int. J. Engng Sci. 3, 47-57.

Timoshenko, S.P., Goodier, J.N., 1970. Theory of Elasticity. 3rd ed. McGraw-Hill, New York.

Way, S., 1940. Some observations on the theory of contact pressures. J. Appl. Mech., Trans. ASME 62, pp. 147-157. 